\title{
One nanoprobe, two pathogens: gold nanoprobes multiplexing for point-of-care
}

\author{
Bruno Veigas ${ }^{1,2}$, Pedro Pedrosa ${ }^{1}$, Fábio F Carlos ${ }^{1,3}$, Liliana Mancio-Silva ${ }^{4}$, Ana Rita Grosso ${ }^{4}$, Elvira Fortunato ${ }^{2}$, \\ Maria M Mota ${ }^{4}$ and Pedro V Baptista ${ }^{1 *}$
}

\begin{abstract}
Background: Gold nanoparticles have been widely employed for biosensing purposes with remarkable efficacy for DNA detection. Amongst the proposed systems, colorimetric strategies based on the remarkable optical properties have provided for simple yet effective sequence discrimination with potential for molecular diagnostics at point of need. These systems may also been used for parallel detection of several targets to provide additional information on diagnostics of pathogens.

Results: For the first time, we demonstrate that a single Au-nanoprobe may provide for detection of two distinct targets (pathogens) allowing colorimetric multi-target detection. We demonstrate this concept by using one single gold-nanoprobe capable to detect members of the Mycobacterium tuberculosis complex and Plasmodium sp., the etiologic agents of tuberculosis and malaria, respectively. Following characterisation, the developed gold-nanoprobe allowed detection of either target in individual samples or in samples containing both DNA species with the same efficacy.

Conclusions: Using one single probe via the non-cross-linking colorimetric methodology it is possible to identify multiple targets in one sample in one reaction. This proof-of-concept approach may easily be integrated into sensing platforms allowing for fast and simple multiplexing of Au-nanoprobe based detection at point-of-need.
\end{abstract}

Keywords: MTBC, Malaria, Nanodiagnostics, Gold nanoparticles, Tuberculosis, rpoB, Plasmodium

\section{Background}

Most molecular diagnostic approaches for pathogen screening have been optimized for single pathogen identification, which is time consuming, costly and rely on highly trained technicians $[1,2]$. Among these, particular emphasis has been brought upon molecular characterization based on PCR amplification and DNA hybridization approaches that detect specific molecular signatures in a few hours, which compare well to classical methods that can take up several days to yield a definite result $[3,4]$. Despite being suitable for centralized wellequipped laboratories, these methodologies fail to deliver when moved to more remote locations and when a fast

\footnotetext{
*Correspondence: pmvb@fct.unl.pt

1 Nanomedicine@FCT, Departamento de Ciências da Vida, Faculdade de Ciências e Tecnologia, CIGMH, UCIBIO, Universidade Nova de Lisboa, Campus de Caparica, 2829-516 Caparica, Portugal

Full list of author information is available at the end of the article
}

screening is required for assistance to clinical protocols. As such, the development of cheap, fast and user-friendly molecular methods at point-of-need is required and would have a huge impact in the capacity of early diagnosis and treatment of pathogen related infections. Fast and accurate results, i.e. early detection, are of utmost importance for the management of patients in terms of pathogen infection spreading and proper implementation of control and treatment measures [2]. Tuberculosis (TB) and malaria are two major global public health threats that undermine development in many resource-poor and some transitional settings with a substantial humanitarian, economic, and social impact $[5,6]$. TB is still one of the leading human infectious diseases responsible for more than 1.5 million deaths and 9 million infections in 2013, together with the increasing rate of multidrugresistant tuberculosis (MDRTB) pose a serious public health problem [7]. Malaria is endemic in 109 countries 
and was responsible for an estimated 207 million cases worldwide in 2012, with an estimated 627000 deaths [6, $8,9]$. As observed with TB, the major burden of malaria occurs in sub-Saharan Africa and Asia. Human co-infection with these two pathogens is quite common [6-8] and represents an important public health problem in coendemic areas of low and middle income countries [6].

Nanotechnology has already provided for powerful tools for molecular diagnostics. Numerous nanoparticlebased approaches have been designed to identify molecular pathogen signatures with extra sensitivity and faster than ever before [10-13]. Nowadays researchers have been gearing their efforts towards the development of nanotechnology-based systems that are affordable, robust and reproducible [14]. As such, nanoparticle based approaches are expected to evolve incrementally over time allowing to meet the needs faced in the field. In particular, systems based on gold nanoparticles (AuNPs) functionalized with thiol-modified DNA (Au-nanoprobes) have been extensively used for the detection and characterization of pathogens [10], including Mycobacterium tuberculosis $[11,12]$. We have previously reported a detection strategy for members of the $M$. tuberculosis complex (MTBC) based on the observable colorimetric alteration of an Au-nanoprobe colloidal suspension [15-18]. The colorimetric alteration results from the differential aggregation profiles of Au-nanoprobes induced by increased ionic strength in the presence or absence of the specific target sequence: presence of the complementary target sequence prevents aggregation and the solution remains red (localized surface plasmon resonance (LSPR) band at $525 \mathrm{~nm}$ ); whereas absence of a specific target sequence leads to nanoparticles aggregation after salt addition and the solution turns blue (red-shift of the LSPR peak to longer wavelength, $600-650 \mathrm{~nm}$ ) [10]. This non-cross-linking method has shown to be extremely sensitive allowing for single point mismatches characterization, and was already applied to the identification of point mutations associated to rifampicin and isoniazid resistance in MTBC [12, 16-18].

Efforts have also been made to extend these system to allow for extra layers of information in a single reaction, either by exploring the differential kinetics of aggregation and hybridization efficiencies or relying on the use of additional plasmon signatures of NPs (e.g. gold:silver alloy nanoprobes) [18-21]. Here, we demonstrate that the $\mathrm{Au}$-nanoprobe approach may be easily extended towards the simultaneous detection of two pathogens of interest with a single multi-sequence functionalized Au-nanoprobe to evaluate in a single test the presence/absence of any of the pathogens. To enhance the detection potential of this approach for MTBC and Plasmodium sp., we performed a preparative multiplex amplification of the respective specific loci-see Fig. 1. This concept extends the colorimetric detection to multiplexing suitable for point-of-need molecular screening.

\section{Methods}

All reagents were purchased from Sigma Aldrich and were of analytical grade. HPLC purified labeled oligonucleotides were purchased from STABVIDA (Portugal) and used without further purification. Thiolated oligonucleotides were used to synthesize the Au-nanoprobes and non-modified oligonucleotides were used as specific controls for calibration of the assay.

\section{Biological samples}

Clinical isolates obtained from respiratory samples positive for acid-fast bacilli (BAAR) from patients of the Lisbon Health Region were used as Tuberculosis positive control (M. tuberculosis H37Rv-ATCC27294 ${ }^{\mathrm{T}}$ ). P. falciparum 3D7 clone were used as Malaria positive control $(P$. falciparum-M19172.1).

\section{Multiplex PCR amplification}

Two conserved regions of MTBC rpoB gene and Plasmodium $18 \mathrm{~s}$ ribosomal RNA (18 s rRNA) were amplified in a single and multiplex PCR reaction using two sets of primer pairs (Additional file 1: Table S1) designed to obtain two different fragments: $395 \mathrm{bp} \mathrm{rpoB}$ and $408 \mathrm{bp}$ $18 \mathrm{~s}$ rRNA. PCR amplifications were performed on a Biometra ${ }^{\circledR}$ TGradient Thermocycler (Göttingen, Germany) in $50 \mu \mathrm{L}$ final volume with $1 \times$ DreamTaq Buffer, $0.1 \mathrm{mM}$ of each DNTPs, $2 \mu \mathrm{M}$ of each primer and $0.1 \mathrm{U}$ $\mu \mathrm{L}$ of DreamTaq DNA polymerase (Amersham Biosciences, GE Healthcare, Europe) and $\sim 1 \mu \mathrm{g} / \mathrm{mL}$ of template DNA with the following thermal cycling conditions: initial 5 min denaturation at $95^{\circ} \mathrm{C}$, followed by 30 amplification cycles of denaturation at $95^{\circ} \mathrm{C}$ for $30 \mathrm{~s}$, annealing at $58^{\circ} \mathrm{C}$ for $30 \mathrm{~s}$, elongation at $72^{\circ} \mathrm{C}$ for $45 \mathrm{~s}$, and a final elongation at $72^{\circ} \mathrm{C}$ for $5 \mathrm{~min}$. PCR products were analyzed in a $1 \%$ agarose gel electrophoresis and quantified via UV/Vis spectroscopy (Nanodrop ND1000, Nanodrop Technologies, USA) (Additional file 1: Figure S1).

\section{Au-nanoprobes design and syntheses}

Au-nanoprobes sequences were designed using Serial Cloner v. 1.3-11 and Geneious v. R7.1 comparative tools aligning the probe sequence with the targeted genes. The multi-functionalized Au-nanoprobe was designed for the detection of two pathogen with two specific sequences in a 1:80:80 (AuNP: oligo1: oligo2) ratio (Additional file 1: Table S1): one sequence complementary to MTBC members [14], and complementary to the $18 \mathrm{~S}$ rRNA gene sequences from Plasmodium sp. For each of the used sequences a single functionalized Au-nanoprobe was also 


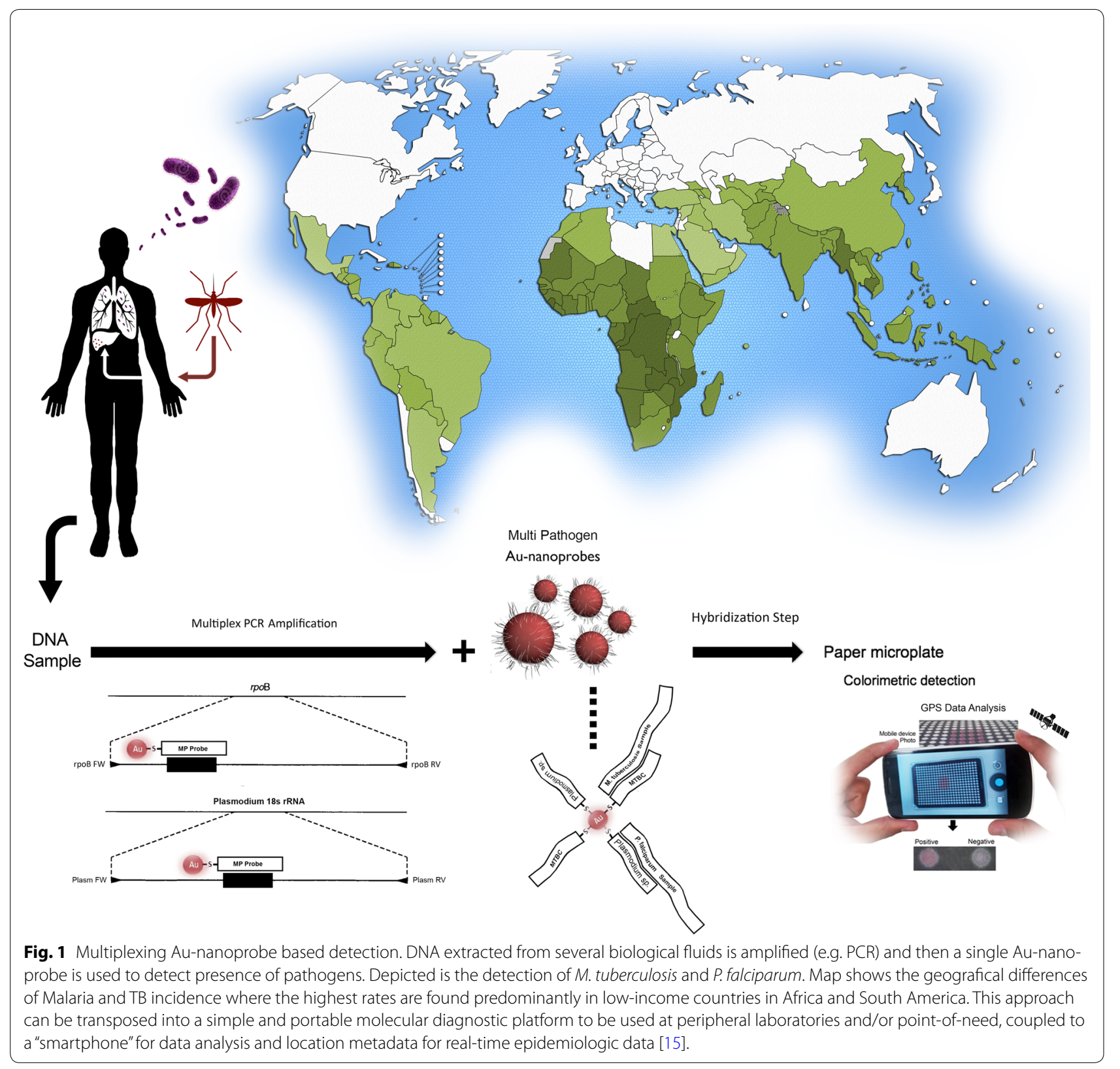

synthesized in a 1:160 (AuNP:oligo) ratio. Gold nanoparticles, with an average diameter of $\sim 14 \mathrm{~nm}$, were synthesized and functionalized as described by Veigas et al. [16] (Additional file 1: Figure S2). Briefly, thiol-modified oligonucleotides were incubated with the AuNPs with increasing salt concentration, in order to reduce non-specific bonds between the thiol-modified oligonucleotides and the AuNPs. After $16 \mathrm{~h}$, the solution was centrifuged, the resulting pellet resuspended in $10 \mathrm{mM}$ phosphate buffer $(\mathrm{pH}), 0.1 \mathrm{M} \mathrm{NaCl}$, and stored in the dark at $4^{\circ} \mathrm{C}$ till further use.

\section{Au-nanoprobe colorimetric assay}

The colorimetric assays were performed in a final volume of $30 \mu \mathrm{L}$ containing Au-nanoprobes at a final concentration of $2.5 \mathrm{nM}$ in $10 \mathrm{mM}$ phosphate buffer $(\mathrm{pH}$ 8 ) and multiplex PCR product at final DNA concentration of $60 \mu \mathrm{g} / \mathrm{mL}$. The mixture was heated up at $95^{\circ} \mathrm{C}$ for $5 \mathrm{~min}$ and then cooled down to $25^{\circ} \mathrm{C}$ for $5 \mathrm{~min}$. For each probe, the assay consisted on the spectrophotometric comparison of a "Blank" (without DNA), $10 \mathrm{mM}$ phosphate buffer ( $\mathrm{pH} 8), 0.1 \mathrm{M} \mathrm{NaCl}$; non-related control containing non-complementary DNA; and the samples. 
The pre-determined $\mathrm{MgCl}_{2}$ concentration was added to each reaction, and after $30 \mathrm{~min}$ at room temperature for color development, the mixtures and the blank assayed by UV/visible spectroscopy in a microplate reader (Tecan Infinite M200). For calibration, each set of $\mathrm{Au}$ nanoprobes was tested against purified simplex PCR amplicons.

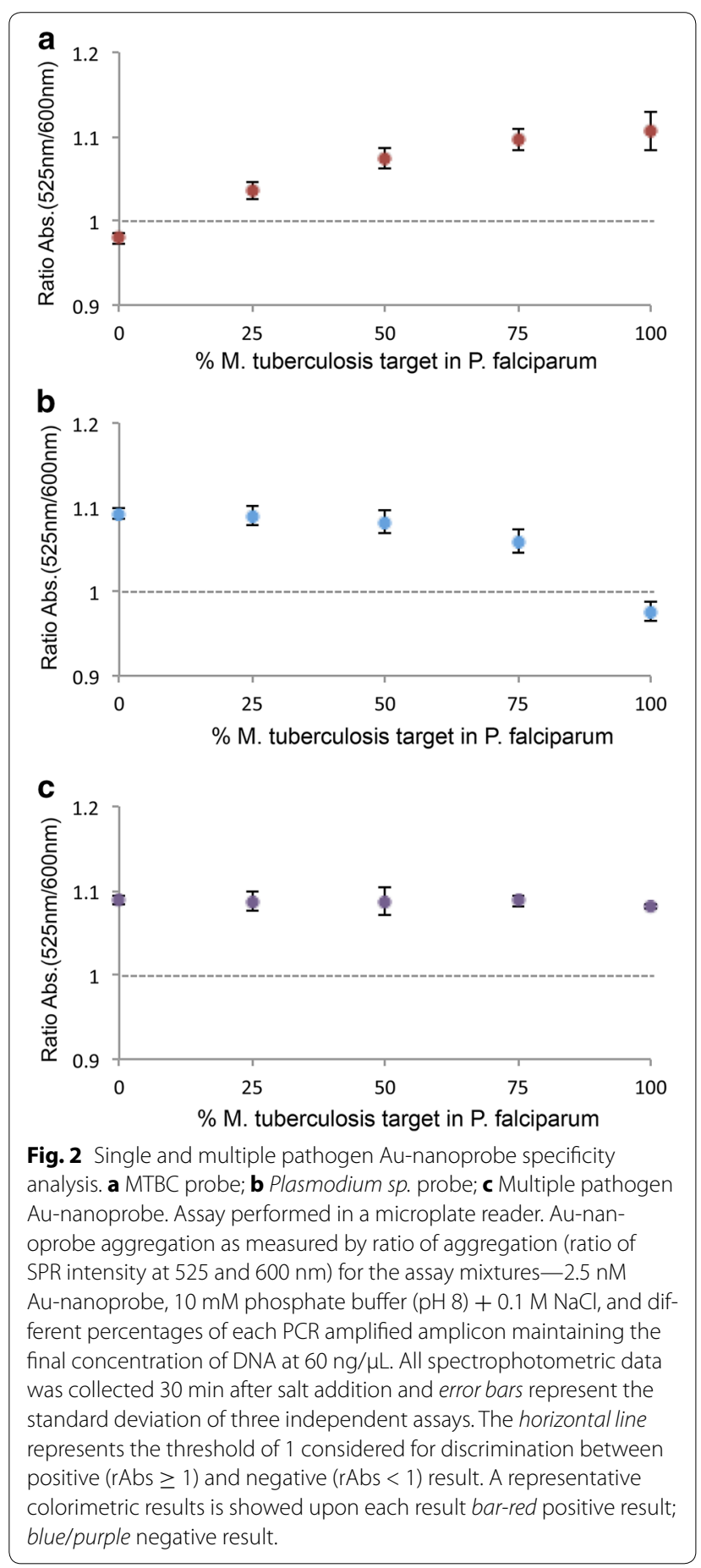

\section{Data analysis}

Aggregation profiles were analyzed in terms of the ratio of $\mathrm{Abs}_{525 \mathrm{~nm}} / \mathrm{Abs}_{600 \mathrm{~nm}}$ (dispersed vs. aggregated species) for each $\mathrm{Au}$-nanoprobe. Each probe was used in a minimum of three individual parallel hybridization experiments with the PCR amplified amplicons derived from the rpoB and $18 \mathrm{~s}$ rRNA genes from MTBC and $P$. falciparum, respectively. A threshold of 1 was considered where values $>1$ indicate that the Au-nanoprobe is mostly non-aggregated (Positive), whereas a value $<1$ indicate aggregation (Negative) [18]. This approach provides for indication of presence or absence of pathogens DNA in the sample.

\section{Results and discussion}

Based on the molecular signatures of pathogens from MTBC and Plasmodium sp., we developed a two-step approach relying on a multiplex PCR amplification and

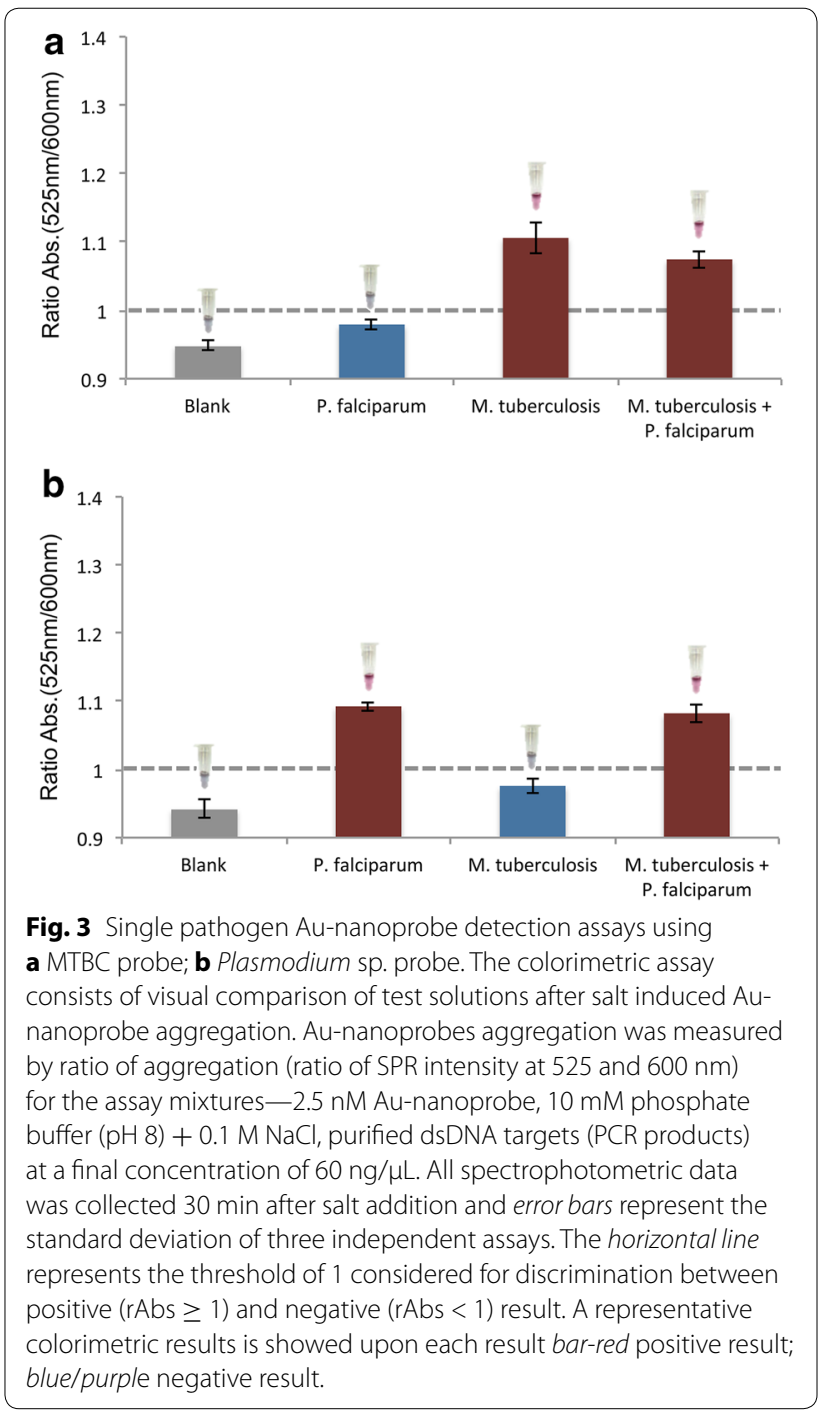


subsequent hybridization with a single Au-nanoprobe. We targeted two loci: rpoB for MTBC and $18 \mathrm{~s}$ rRNA genomic sequences for Plasmodium sp. The specific set of primer allow amplification of unique genomic regions for strains belonging to these sub-groups and constitute the first line of selective detection, then the Au-nanoprobe assay a serves as the second line of identification (see Fig. 1). The presented method relies on the differential aggregation profile of Au-nanoprobes following an increase to ionic strength of the medium (e.g. salt addition, $\mathrm{MgCl}_{2}$ ). Hybridization to the complementary target stabilizes the $\mathrm{Au}$-nanoprobe from the induced aggregation and the solution remains red; whereas no hybridization (no complementary target) leads to extensive aggregation and concomitant red-shift of the plasmon band and the solution turns blue [18]. The two loci were selected to allow for discriminatory detection of human pathogens: $r p o B$ primers and MTBC probe specifically recognize members of the $M$. Tuberculosis complex; detection of $18 \mathrm{~s}$ rRNA genomic sequences for the most common species of parasites, P. Falciparum, P. Vivax, P. malariae, and P. Ovale [8]. The latter is also capable to recognize other more uncommon species, such as $P$. knowlesi and primate infecting type Plasmodium cynomolgi, with a recent reported case of natural human infection [22]. This screening approach assures that any of the species capable of infecting humans are amplified and detected by the nanoprobe assay.

First, we optimized the multiplex PCR amplification so that both two pathogens' gene fragments are present with similar yields while maintaining PCR specificity.
Primers were designed so as to produce amplicons showing similar sizes, since comparable hybridization efficiencies and profiles are desired [18]. The two loci were successfully amplified alone and then in a single multiplex reaction from DNA isolates of the pathogens (Additional file 1: Figure S1). Then, each Au-nanoprobe sequence was evaluated in terms of selectivity and specificity towards the respective target in solution (Fig. 2). Calibration of the Au-nanoprobe functionalization in presence of the respective target, but aggregated in its absence (see Additional file 1: Figures S3 and S4). No noteworthy loss in signal occurs when both pathogens amplicons are present in the mixture compared to each amplicon alone (Fig. 3).

The principle of a multiple functionalized Au-nanoprobe relies on the simultaneous functionalization of AuNPs with specific oligonucleotides for both target sequences in a 1:80:80 (AuNP: oligo1: oligo2) ratio. This $\mathrm{Au}$-nanoprobe was able to detect each target sequence individually in solution and when the target is in a multiplex PCR mixture (Figs. 3, 4).

\section{Conclusion}

There is a continuous and strenuous demand for robust, yet simple molecular diagnostics capable to identify pathogens at point-of-care. The lack of laboratorial infrastructures in places at the forefront of disease areas, where simultaneous infections often occur, have been showing the relevance of developing simple assays that may provide immediate responses to the clinicians. Here, for the first time, the potential of using a single Au-nanoprobe

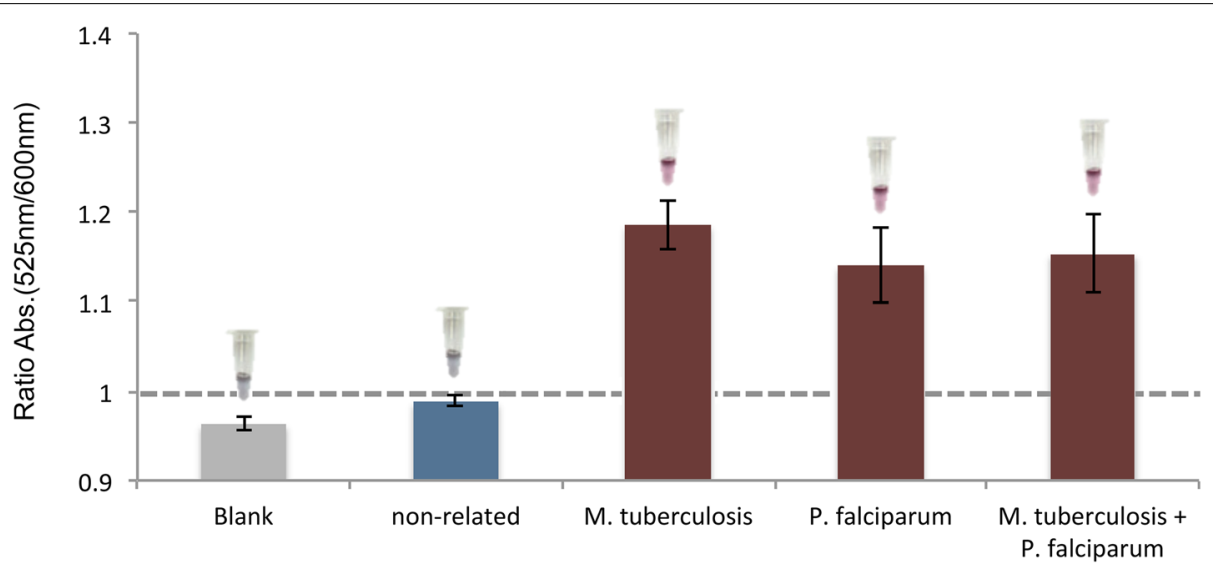

Fig. 4 One probe, two pathogens assay. Use of a single Au-nanoprobe for sample characterization harbouring a single target (M. tuberculosis or $P$. falciparum) and both targets. The amount of total DNA in each sample is constant $(60 \mathrm{\mu g} / \mathrm{mL})$. Au-nanoprobes aggregation was measured by ratio of aggregation (ratio of SPR intensity at 525 and $600 \mathrm{~nm}$ ) for the assay mixtures-2.5 nM Au-nanoprobe, $10 \mathrm{mM}$ phosphate buffer (pH 8) $+0.1 \mathrm{M}$ $\mathrm{NaCl}$, purified dsDNA targets (PCR products) at a final concentration of $60 \mathrm{ng} / \mathrm{\mu L}$. All spectrophotometric data was collected $30 \mathrm{~min}$ after salt addition and error bars represent the standard deviation of three independent assays. The horizontal line represents the threshold of 1 considered for discrimination between positive $(\mathrm{rAbs} \geq 1)$ and negative $(\mathrm{rAbs}<1)$ result. A representative colorimetric results is showed upon each result bar-red positive result; blue/purple negative result. 
for the specific detection of multiple targets in a single reaction is demonstrated. A two-step approach was designed based on the amplification of DNA samples followed by detection with a single Au-nanoprobe for the simultaneous specific detection of M. tuberculosis and P. falciparum, without loss of sensitivity. Due to its simplicity, this approach may easily be translated to remote areas. Nevertheless, under the present format, a thermocycler and spectrophotometer are still required but alternatively isothermal amplification [17] and cheap disposable platform [15] may eliminate the need of such need equipment with comparable results. The concept here presented may easily be extended to further pathogens and targets aiming at cost reduction and enlarge the range of molecular testing for pathogens at point of need.

\section{Additional file}

Additional file 1: TableS1. Multiplex PCR primers and Au-nanoprobes sequences. Figure S1. Electrophoretic analysis on 1\% agarose gel: In lane 1 was used Thermo Scientific GeneRuler DNA Ladder Mix; Lane 2 and 3 Multiplex PCR products of M. tuberculosis and P. falciparum, respectively; Lane 4 Multiplex PCR product of M. tuberculosis and P. falciparum, Lane 5 negative control of the PCR reaction. Figure S2. Physical characterization of the synthesized gold nanoparticles. A) Transmission electron microscopy (TEM) imaging and inset size histogram frequency from $\approx$ 400 AuNPs counting; B) UV-Vis spectrum of spherical AuNPs, with a characteristic maximum absorption peak, C) Dynamic light scattering (DLS) measurements to define the hydrodynamic diameter of the AuNPs and zeta-potential ( $\zeta$-potential) as AuNP surface charge indicator. For DLS it was performed 3 runs per sample with 2000 measurements each and for $\zeta$-potential a total of 5 runs per sample with 250 measurements. Figure S3. Aggregation profiles of the synthesized Au-nanoprobes. Aggregation measured as the ratio of localized surface plasmon resonance intensity at $525 \mathrm{~nm}$ and $600 \mathrm{~nm}$ for increasing salt concentrations $\left(\mathrm{MgCl}_{2}\right)$. The minimum amount of salt required to cause aggregation was determined based on each Au-nanoprobe aggregation profiles. Ratio $<1$ was considered for full aggregation, $60 \mathrm{mM}$. Figure $\mathbf{S 4}$. Single pathogen Au-nanoprobe specificity analysis using synthetic targets. A 1 pmol concentration of synthetic targets (see Table S1) were individually tested with a $\mathrm{MgCl}_{2}$ concentration of $60 \mathrm{mM}$.

\section{Abbreviations}

AuNPs: gold nanoparticles; LSPR: localized surface plasmon resonance; MDRTB: multi-drug resistant tuberculosis; MTBC: Mycobacterium tuberculosis complex; TB: tuberculosis.

\section{Authors' contributions}

FC synthesized and characterized the Au-nanoprobes. BV and PP performed the multiplex PCR and the detection assays. LM and AG characterized the biological samples through standard diagnostic methodologies. PB, EF and MM conceived the study, participated in its design and coordination, and drafted the manuscript. All authors read and approved the final manuscript.

\section{Author details}

${ }^{1}$ Nanomedicine@FCT, Departamento de Ciências da Vida, Faculdade de Ciências e Tecnologia, CIGMH, UCIBIO, Universidade Nova de Lisboa, Campus de Caparica, 2829-516 Caparica, Portugal. ${ }^{2}$ Departamento de Ciência dos Materiais, Faculdade de Ciências e Tecnologia, CENIMAT/I3N, Universidade Nova de Lisboa, Caparica, Portugal. ${ }^{3}$ STABVIDA, Investigação e Serviços em Ciências Biológicas, Lda. Madan Parque, 2825-182 Caparica, Portugal. ${ }^{4}$ Instituto de Medicina Molecular, Universidade de Lisboa. Av. Prof. Egas Moniz, 1649-028 Lisbon, Portugal.

\section{Acknowledgements}

We acknowledge Fundação para a Ciência e a Tecnologia (FCT-MEC) for financial support CIGMH (PEst-OE/SAU/UI0009/2013) and PTDC/BBBNAN/1812/2012; SFRH/BD/78970/2011 to BV, SFRH/BDE/51103/2010 to FC, EXCL/IMI-MIC/0056/2012 to MMM and PTDC/SAU-MET/118199/2010 to LMS. We also acknowledge funding from the European Research Council under grant agreement no 311502 (MMM) M. Viveiros and I. Couto for M. tuberculosis sample DNA. C. Oliveira for technical support. We also thank Santander-Totta/ UNL for financial support (Scientific Prize Edition 2012).

\section{Compliance with ethical guidelines}

\section{Competing interests}

The authors declare that they have no competing interests.

Received: 28 March 2015 Accepted: 9 July 2015

Published online: 07 August 2015

\section{References}

1. Parsons LM, Somoskövi Á, Gutierrez C, Lee E, Paramasivan CN, Al Abimiku et al (2011) Laboratory diagnosis of tuberculosis in resource-Poor countries: challenges and opportunities. Clin Microbiol Rev 24:314-350

2. Dorman SE (2010) New diagnostic tests for tuberculosis: bench, bedside, and beyond. Clin Infect Dis 50:S173-S177

3. Tang YW, Procop GW, Persing DH (1997) Molecular diagnostics of infectious diseases. Clin Chem 43:2021-2038

4. Viveiros M, Martins M, Couto I, Rodrigues L, Machado D, Portugal I et al (2010) Molecular tools for rapid identification and novel effective therapy against MDRTB/XDRTB infections. Expert Rev Anti Infect Ther. 8:465-480

5. Vitoria M, Granich R, Gilks CF, Gunneberg C, Hosseini M, Were W et al (2009) The global fight against HIV/AIDS, tuberculosis, and malaria. Am J Clin Pathol 131:844-848

6. Wiwanitkit V (2006) Co-infection between tuberculosis and malaria: a consideration on interaction of molecules and pathogenesis. J Vector Borne Dis 43:195-197

7. World Health Organization (2014) Global Tuberculosis Report 2014. WHO, Geneva

8. Word Health Organisation (2013) World Malaria Report 2013. WHO, Geneva

9. Shapiro HM, Perlmutter NG (2008) Killer applications: toward affordable rapid cell-based diagnostics for malaria and tuberculosis. Cytom B Clin Cytom 74:152-164

10. Radwan S, Azzazy H (2009) Gold nanoparticles for molecular diagnostics. Expert Rev Mol Diagn 9:511-524

11. Veigas B, Doria G, Baptista PV (2012) Nanodiagnostics for tuberculosis. In: Cardona PJ (ed) Understanding tuberculosis_-global experiences and innovative approaches to the diagnosis, chap 12. InTech, Croatia, pp 257-276. ISBN 979-953-307-078-9. Available from: http://www.intechopen.com/books/understanding-tuberculosisglobal-experiences-and-innovative-approaches-to-the-diagnosis/ nanodiagnostics-for-tuberculosis

12. Veigas B, Fernandes AR, Baptista PV (2014) AuNPs for identification of molecular signatures of resistance. Front Microbiol 5:455

13. Zeng S, Yong KT, Roy I, Dinh XQ, Yu X, Luan F (2011) A review on functionalized gold nanoparticles for biosensing applications. Plasmonics 6:491-506

14. Gauglitz G (2014) Point-of-care platforms. Annu Rev Anal Chem 7:297-315

15. Veigas B, Jacob JM, Costa MN, Santos DS, Viveiros M, Inacio J et al (2012) Gold on paper-paper platform for Au-nanoprobe TB detection. Lab Chip 12:4802-4808

16. Veigas B, Machado D, Perdigao J, Portugal I, Couto I, Viveiros M et al (2010) Au-nanoprobes for detection of SNPs associated with antibiotic resistance in Mycobacterium tuberculosis. Nanotechnology 21:415101

17. Veigas B, Pedrosa P, Couto I, Viveiros M, Baptista PV (2013) Isothermal DNA amplification coupled to Au-nanoprobes for detection of mutations associated to Rifampicin resistance in Mycobacterium tuberculosis. J Nanobiotechnol 11:38 
18. Pedrosa P, Veigas B, Machado D, Couto I, Viveiros M, Baptista PV (2014) Gold nanoprobes for multi loci assessment of multi-drug resistant tuberculosis. Tuberculosis 94:332-337

19. Doria G, Larguinho M, Dias JT, Pereira E, Franco R, Baptista PV (2010) Gold-silver-alloy nanoprobes for one-pot multiplex DNA detection. Nanotechnology 21:255101

20. Elenis D, loannou P, Christopoulos T (2011) A nanoparticle-based sensor for visual detection of multiple mutations. Nanotechnology 22:155501
21. Seowa N, Laib PS, Yung LY (2014) Gold nanostructures for the multiplex detection of glucose-6-phosphate dehydrogenase gene mutations. Anal Biochem 451:56-62

22. Ta TH, Hisam S, Lanza M, Jiram Al, Ismail N, Rubio JM (2014) First case of a naturally acquired human infection with Plasmodium cynomolgi. Malar J 13:68

Submit your next manuscript to BioMed Central and take full advantage of:

- Convenient online submission

- Thorough peer review

- No space constraints or color figure charges

- Immediate publication on acceptance

- Inclusion in PubMed, CAS, Scopus and Google Scholar

- Research which is freely available for redistribution

Submit your manuscript at www.biomedcentral.com/submit 